\title{
Impairment of spatial performance by continuous intensive noise: a behavioral view
}

\author{
Mohammad Javad Deylam ${ }^{1}$, Mohammad Ali Gheraat ${ }^{2}$, Nasser Naghdi ${ }^{3}$ and Leila Eidi Abarghani ${ }^{2}$ \\ 1 - Islamic Azad University, Ali Abad Katool Branch, Iran \\ 2 - Islamic Azad University, Islamshahr Branch, Iran \\ 3 - Pasteur Institute of Iran, Tehran, Iran
}

\begin{abstract}
A wide range of animal and human investigations have tested the neurobiological and immunological aspects of noise. Few studies, however, have explored the behavioral characteristics of noise on neuromotor movements. To examine this correlation, we tested the effects of continuous intensive noise on retention and contextual transfer in a spatial memory task in adult male rats. The natural noise was recorded in a football stadium and set at high (HI), moderate (MI), and low (LI) intensities, levels corresponding to 86-90, 64-68, and 52-54 A-weighted decibels (dbA), respectively. Rats were trained in a Morris water maze for 3 consecutive days. On day 4, visible and probe tests were conducted under the same intensities. Retention was evaluated on day 7 with high-intensity noise exposure. The contextual transfer test was held on day 8 after exposure to 30-min highintensity noise. The escape latency and distance traveled were recorded and used for subsequent analyses. Our results showed significant increases in latency and distance traveled, attributable to increasing the noise intensity during the acquisition period. Additionally, performance in the LI group was significantly impaired in the retention test at the high intensity. In the contextual transfer test, results showed no significant increase except in the LI group, whereas a higher latency and distance traveled were found in the HI group. High-intensity noise appeared to damage the learning process. However, because the most robust results were found in the MI group, training with moderate-intensity noise can promote better performance under continuous highintensity noise. Keywords: continuous intensive noise, retention, contextual transfer, spatial memory task, rat.
\end{abstract}

Received 6 November 2011; received in revised form 12 December 2011; accepted 15 December. Available online 29 December 2011

\section{Introduction}

One aspect of the extensive range of activities that can be affected by different types of noise (e.g., low or high intensity, impulsive or continuous noise) is task performance. A wide range of previous animal and human studies of the effects of noise have been performed mainly within the framework of the neurobiological, psycho-immunological, and developmental fields (Maes $\&$ de Groot, 2002). However, new investigations have been conducted to study the positive and negative effects of noise on behavioral characteristics, contributing

Mohammad Javad Deylam, Faculty of Physical Education \& Sport Sciences, Islamic Azad University, Ali Abad Katool Branch, Iran. Mohammad Ali Gheraat and Leila Eidi Abarghani, Faculty of Physical Education \& Sport Sciences, Islamic Azad University, Islamshahr Branch, Iran. Nasser Naghdi, Department of Physiology \& Pharmacology, Pasteur Institute of Iran, Tehran, Iran. Correspondence regarding this article should be directed to: Mohammad Ali Gheraat, Islamic Azad University, Islamshahr Branch, Faculty of Physical Education \& Sport Sciences. Address: Faculty of Sport Sciences, 6th Alley, Takhti Street, Islamshahr, Iran. E-mail: mohammadaligharaat@gmail.com to our understanding of the variable effects of high-, medium-, and low-intensity noise. Some investigations have found that tasks are affected by exposure to unpredictable and uncontrollable high-intensity noise. This factor is assumed to result in learned helplessness, cognitive fatigue, and a consequent loss of information processing capacity or even overarousal (Glass \& Singer, 1972; Yerkes \& Dodson, 1908). The effects of these impairments on verbal and behavioral reactions (Fiedler \& Fiedler, 1975), the amplitude and latency of performance (Dimitrijevic, Michalewski, Zeng, Pratt, \& Starr, 2008), spatial performance and learning (Gheraat et al., 2009), behavioral and pathological changes in diving mammals (Jepson et al., 2003; Malakoff, 2001; Talpalery \& Grossman, 2005), psychomotor and learning tasks (Broadbent, 1979; Schmidt, 1999), and sports referees' decisions (Balmer, Nevill, Lane, Ward, Williams, \& Fairclaugh, 2007; Nevill, Balmer, \& Williams, 2002) are highly variable. In contrast, exposure to an appropriate amount of noise improves signal detection in stochastic resonance (Zeng, Fu, Morse, 2000), human hearing (Maes \& de Groot, 2002), and the ability to encode temporal information (Maes \& 
de Groot, 2002; Waye, 2003). Another study noted that noise during testing can either enhance or decrease task performance, depending on the noise intensity during training (Bell, Hess, Hill, Kukas, Richards, \& Sargent, 1984). Other research showed that performance on a verbal memory task was impaired after training under a different intensity of noise unequal to the noise intensity during the training period.

In addition to the above studies, some research has focused on the effects of intermittent $v s$. continuous noise on performance, but results have been variable. A wide range of noise intensities in general and the complexity of the tasks in particular may be reasons for this variation (Broadbent, 1979; Hambrick-Dixon, 1986; Schmidt, 1999). A study in a day-care center showed that children exposed to long-term noise performed best under a noisy test condition, whereas children exposed to a quiet training context performed best under a silent contextual retention test (Hambrick-Dixon, 1986). The findings indicated that adaptation to the training context may be a main reason for the correlation. In another investigation, listeners experienced better perceptual performance under interrupted noise than they did under continuous noise at equivalent signal-to-noise ratios (Stuart, 2005). In contrast, a similar reduction in the amplitude of evoked potentials was found after both continuous and intermittent noise exposure. However, latency-intensity functions of the individual waves of potentials evoked along the auditory pathway did not change compared with the same click intensity before and after exposure to high-intensity noise (Syka \& Popelar, 1982). Another study showed that task-related components such as working memory and motor feedback response increased for continuous noise as opposed to impulsive noise (Haller, Homola, Scheffler, Beckmann, \& Bartsch, 2009). Another investigation in which rats performed water maze tasks while being exposed to $80 \mathrm{db}$ sound pressure level (SPL) interrupted white noise for 2 weeks found continuous improvement in performance in the control group and in rats exposed to noise during training, whereas rats exposed to noise at a young age exhibited significantly poorer performance in adulthood compared with rats not exposed to noise at a young age (Zhang, Chen, Gao, Pu, \& Sun, 2008). Additionally, the effects of $120 \mathrm{db}$ SPL continuous noise compared with intermittent noise were more damaging to the vestibular system of guinea pigs, which plays a vital role in memory (Akdogan, Selcuk, Take, Erdoğan, \& Dere, 2009).

However, effects of continuous high-intensity noise on the contextual transfer test after applying various intensities of noise during training have not been investigated. The importance of matching training and test conditions may be confirmed by the results of the former studies (Gheraat et al., 2009; Maes \& de Groot, 2002). These findings may be expanded with the results of the present study. Therefore, the main purpose of the present study was to test the effects of continuous highintensity noise on the learning (i.e., acquisition, retention, and transfer) of spatial memory tasks depending on the intensity of noise during the training period.

\section{Methods}

\section{Animals}

Thirty-two adult male albino rats (12 weeks old) weighing 250-280 $\mathrm{g}$ were obtained from the breeding colony of the Pasteur Institute of Iran. All experimental procedures were in accordance with the animal care guidelines outlined by the Animal Care Committee of the Pasteur Institute of Iran (Tehran, Iran). In each plastic cage were either three or four adult rats maintained on a $12 \mathrm{~h} / 12 \mathrm{~h} \mathrm{light/dark}$ cycle (lights at 7:00 AM). All rats had free access to food and water. The ambient temperature was maintained at $20-24^{\circ} \mathrm{C}$. Experiments were conducted in a room where only the water maze was placed under standard conditions and occurred between 9:00 AM and 11:00 AM.

\section{Water maze apparatus}

The Morris water maze task consisted of a dark circular pool $(140 \mathrm{~cm}$ diameter, $55 \mathrm{~cm}$ height) filled with water $\left(20 \pm 1^{\circ} \mathrm{C}\right)$ to a depth of $25 \mathrm{~cm}$. A transparent Plexiglas platform (11 cm diameter) was located $1 \mathrm{~cm}$ below the water surface in the center of one of the arbitrarily designed northeast (NE), northwest (NW), southwest (SW), or southeast (SE) orthogonal quadrants. The platform provided the only escape from the water. Extra-maze cues such as racks, a window, a door, book shelves, a clock, and pictures on the walls were in the surrounding environment where the water maze was housed. These cues were kept in fixed positions with respect to the swimming pool to allow the rat to locate the escape platform hidden below the water surface. A video tracking system and personal computer with software developed for monitoring and storing the position of the rat in the water maze were used. Thus, the time required to reach the platform (latency) and swimming path (distance) were recorded (Naghdi, Rezaei, \& Fathollahi, 2006).

\section{Noise regulation}

The intensity of noise was calibrated from a football stadium that contained $\sim 100,000$ spectators using a Sound Level Meter (B\&K 2250). Intensity of noise at the high intensity was $\sim 105 \mathrm{dbA}$. The intensity of noise was $\sim 95-98 \mathrm{dbA}$ in the moderate-intensity condition and $69-72 \mathrm{dbA}$ in the silent condition. First we recorded this natural sound from the stadium. The sound levels were then reduced to adjust to the hearing system of rats (Downey \& Harrison, 1992). They were reduced to 8690, 64-68, and 52-54 dbA for high, moderate, and low intensities of noise, respectively. The acoustic system 
was regulated according to these intensities with a stable frequency set in Cool-Edit 2000 Software (Emperor Company). The background sound level of the room where the rats were reared under standard conditions was $47 \mathrm{dbA}$ in which the noise emanated from an airconditioner and other environmental sound sources. The previously mentioned noises were presented with two loudspeakers situated $150 \mathrm{~cm}$ from the rats.

\section{Procedure}

The spatial memory task involved finding a hidden platform in the water maze. All rats were given a daily session of two blocks of trials, with each block consisting of four trials and 5-min intervals between blocks for 3 days during the training period. During all of the experiments, the escape platform was located in a fixed position in the middle of the SW quadrant. Each trial involved placing the rat into the pool close to the wall and orientating it toward one of the four equal quadrants into which the pool was divided. Animals were allowed to swim freely until they found the escape platform. If a rat failed to find the platform within 90 $\mathrm{s}$, then the experimenter placed it on the platform. The intertrial interval was $30 \mathrm{~s}$ since the rat remained on the platform. The rat was taken directly from the platform to the new starting position, which was changed from trial to trial in a quasi-random order so that each starting point was used once in each session of four trials. Rats were divided into four groups. Control group (control) was trained under natural laboratory noise (47 dbA; $n=8$ ). Low-intensity noise group (LI) was trained under the natural noise of daily life $(52-54 \mathrm{dbA} ; n=8)$. Moderate-intensity noise (MI) and high-intensity noise (HI) groups were trained under 64-68 and 86-90 dbA noise intensities, respectively ( $n=8$ per group). For the first 3 consecutive days, rats were trained in the Morris water maze while being exposed to noise. On day 4 , probe and visible tests were performed. For the probe test, the platform was removed from the water maze and rats were allowed to swim and search for the platform and the SW quadrant. Sixty minutes after the probe test, the platform was covered with aluminum foil and placed $1 \mathrm{~cm}$ above the water surface in the SE quadrant, and the visible test was conducted. This procedure is believed to provide information about the possible nonspecific effects on motor and visual abilities (Gheraat et al., 2009; Naghdi et al., 2006). On day 7, the retention test was performed with the platform placed in the SW quadrant. This test was similar to the training day procedure with regard to blocks and number of trials per session, except that all groups were exposed to high-intensity noise. Twenty-four hours later (day 8), the contextual transfer test was performed similar to the training day procedure immediately after the rats were exposed to high-intensity noise for $30 \mathrm{~min}$, which was the average time recorded at several athletic games such as volleyball.

\section{Statistical analysis}

Data were analyzed using two-way analysis of variance (ANOVA) to assess intergroup data followed by the Tukey post hoc test. Intragroup data were also analyzed by repeated-measures ANOVA. All results are expressed as mean \pm SEM. In all of the statistical comparisons, $p<0.05$ was considered statistically significant. Data were analyzed using SPSS version 13 software.

\section{Experimental procedure}

Stage 1. The aim of this experiment was to determine the effect of control, LI, MI, and HI noise during the acquisition phase of the Morris water maze task. In this experiment, rats were trained with four intensities (control, LI, MI, and HI) of noise in the training period according to the above procedure (3 days of training).

Stage 2. Visible and probe tests were held on day 4 according to the above procedure. The aim of these tests was to determine the effects of noise on motor and visual abilities.

Stage 3. The aim of this experiment was to determine the effect of high-intensity noise on the retention test of the Morris water maze task. In this experiment, rats that had been trained with all four intensities of noise (control, LI, MI, and HI) during the training period were tested for high-intensity noise performance according to the above procedure (3 days of training).

Stage 4. The aim of this experiment was to determine the effect of continuous high-intensity noise on Morris water maze performance in the retention test. In this experiment, the rats that had been trained with all four intensities of noise (control, LI, MI, and HI) during the training period were tested under high-intensity noise immediately after being exposed to $30 \mathrm{~min}$ of high-intensity noise according to the above procedure (3 days of training).

\section{Results}

Data were acquired from the acquisition period and retention tests with common and continuous exposure to high-intensity noise. The escape latency (Tables $1 \mathrm{~A}$ and $1 \mathrm{~B}$ ) and distance traveled (Tables $2 \mathrm{~A}$ and $2 \mathrm{~B}$ ) were recorded. In all experiments, swim speed was not significantly different. Therefore, swim speed results are not presented here.

\section{Training period and visible test}

\section{Hidden platform trials (day 1 to 3 intragroup)}

Two-way ANOVA revealed significant effects of noise intensity on both time and distance $\left(F_{2,28}=84.22\right.$, 
$p<.001$, and $F_{2,28}=78.61, p<.001$, respectively). As shown in Figure $1 \mathrm{~A}$, significant noise intensity $\mathrm{x}$ group interactions were observed for both factors $\left(F_{6,28}=\right.$ $3.74, p=.003$, and $F_{6,28}=4.24, p=.001$, respectively). Regarding the intragroup analysis, repeated-measures ANOVA revealed a significant $(p<.05)$ main effect of training on performance in the Morris water maze task. In the control group, a significant difference (latency: $\Lambda=0.05, F_{3,5}=16.42, p=.02$; distance: $\Lambda=0.04, F_{3,5}$ $=12.22, p=.03)$ was found between day 1 and days 2 and $3(p<.05)$. In the LI group, a significant difference (latency: $\Lambda=0.03, F_{3,5}=14.87, p=.02$; distance: $\Lambda=$ $\left.0.04, F_{3,5}=12.76, p=.03\right)$ was found between day 1 and days 2 and $3(p<.05)$ when escape latency and the distance traveled gradually decreased. In the MI group, a significant difference (latency: $\Lambda=0.07, F_{3,5}=15.26$, $p=.04$; distance: $\Lambda=0.06, F_{3,5}=10.79, p=.04$ ) was found between day 1 and days 2 and $3(p<.05)$, which was same in the control and LI groups (Figure 1A). Ultimately, the findings from the HI group showed the same results (latency: $\Lambda=0.03, F_{3,5}=19.32, p=$

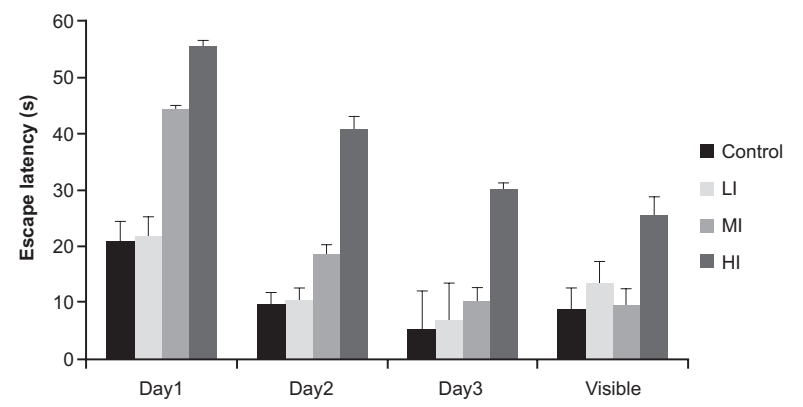

.01 ; distance: $\Lambda=0.04, F_{3,5}=11.77, p=.03$ ). In this group, a significant difference was found between day 1 and days 2 and $3(p<.05)$ in escape latency. The analysis also revealed a significant difference between day 1 and day $3(p<.05)$ in distance traveled (Figure $2 \mathrm{~A})$. The main decrease in these two factors occurred between day 1 and day 2 of the training period.

\section{Visible and probe trials (day 4)}

Significant differences were found in performance between groups in the visible platform trials with regard to escape latency $\left(F_{3.28}=13.54, p<.05\right)$ and distance traveled $\left(F_{3,28}=13.79, p<.05\right)$. With regard to escape latency (Figure 1A), significant differences were found between the control, LI, and MI groups and HI group ( $p$ $<.05$ ). As shown in Figure 2A, a significant difference was found between the Control, LI, and MI groups and HI group $(p<.05)$ in distance traveled. With regard to the probe test results (Figure 3), the time searching for the SW quadrant in the HI group was significantly different from the other groups $\left(F_{3,28}=11.08, p<.05\right)$.

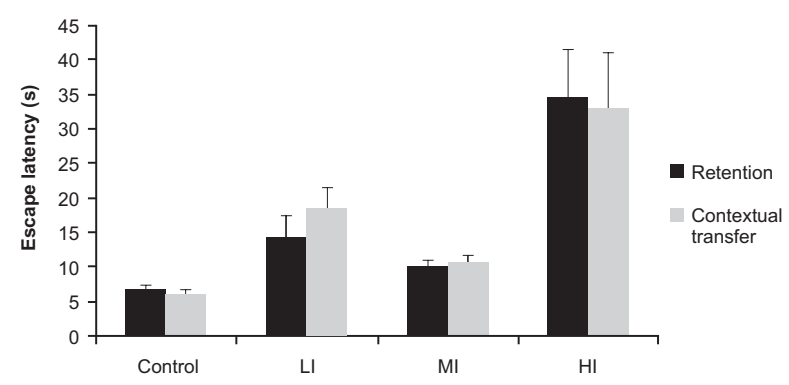

Figure 1. (A) Effects of various intensities of noise during the training period on escape latency on days 1-3 and day 4 (visible test). (B) Differences in escape latency in the retention test with exposure to intermittent high-intensity noise and the contextual transfer test with exposure to continuous noise.

Table 1A. Descriptive results of latency in the training period and visible test

\begin{tabular}{|c|c|c|c|c|c|c|c|c|}
\hline \multirow[t]{2}{*}{ Latency } & \multicolumn{2}{|c|}{ Day 1} & \multicolumn{2}{|c|}{ Day 2} & \multicolumn{2}{|c|}{ Day 3} & \multicolumn{2}{|c|}{ Visible } \\
\hline & Mean & $\mathrm{SE}$ & Mean & SE & Mean & SE & Mean & $\mathrm{SE}$ \\
\hline Control & 20.92 & 3.58 & 9.64 & 1.53 & 5.33 & 0.5 & 8.7 & 0.81 \\
\hline LI & 21.7 & 2.27 & 10.41 & 2.1 & 6.88 & 1.57 & 13.43 & 2.04 \\
\hline MI & 44.51 & 6.64 & 18.61 & 3.06 & 10.35 & 2.28 & 9.48 & 0.89 \\
\hline HI & 55.71 & 3.85 & 40.86 & 2.86 & 30.25 & 3.2 & 25.41 & 3.45 \\
\hline
\end{tabular}

Table 1B. Descriptive results of latency in retention and transfer tests

\begin{tabular}{lllll} 
Latency & \multicolumn{2}{c}{ Contextual transfer } \\
\cline { 2 - 5 } & Mean & SE & Mean & SE \\
Control & 6.64 & 0.72 & 6.04 & 0.63 \\
LI & 14.37 & 3 & 18.56 & 2.86 \\
MI & 10.00 & 0.92 & 10.75 & 0.87 \\
HI & 34.50 & 6.94 & 32.99 & 7.97 \\
\hline
\end{tabular}



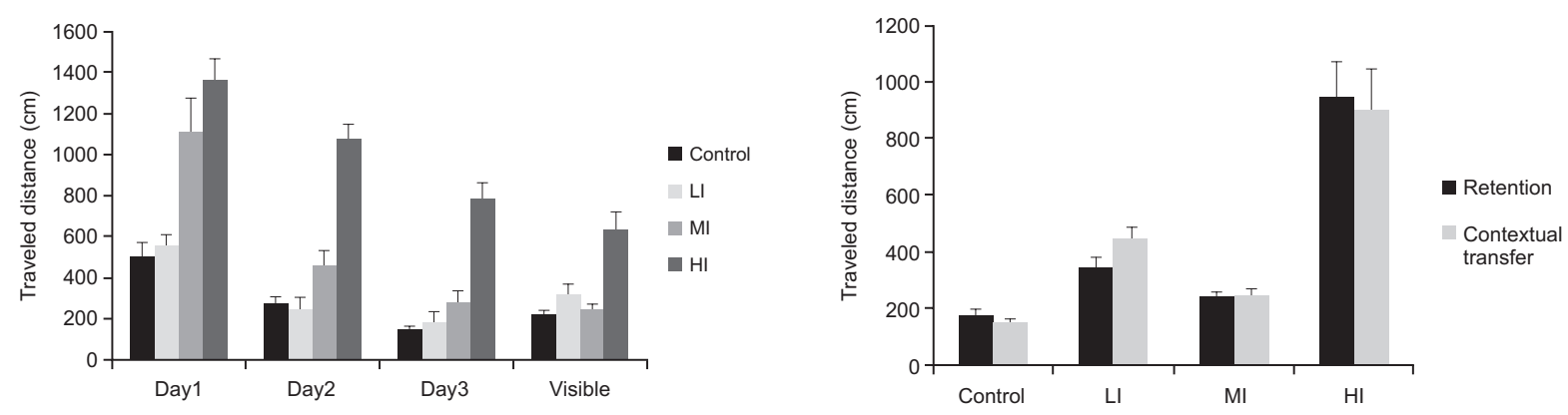

Figure 2. (A) Effects of various intensities of noise during the training period on distance traveled on days 1-3 and day 4 (visible test). (B) Differences in distance traveled in the retention test with exposure to intermittent high-intensity noise and contextual transfer test with exposure to continuous noise.

Table 2A. Descriptive results of distance traveled in the training period and visible test

\begin{tabular}{|c|c|c|c|c|c|c|c|c|}
\hline \multirow{2}{*}{ Distance } & \multicolumn{2}{|c|}{ Day 1} & \multicolumn{2}{|c|}{ Day 2} & \multicolumn{2}{|c|}{ Day 3} & \multicolumn{2}{|c|}{ Visible } \\
\hline & Mean & $\mathrm{SE}$ & Mean & SE & Mean & SE & Mean & SE \\
\hline Control & 495.56 & 72.97 & 266.25 & 39.26 & 143.87 & 12.93 & 218.87 & 21.09 \\
\hline LI & 552.18 & 53.89 & 249.43 & 50.56 & 185.18 & 48.22 & 320.02 & 47.16 \\
\hline MI & 1110.31 & 165.81 & 462.18 & 69.3 & 279.62 & 54.89 & 246.18 & 25.37 \\
\hline HI & 1364.68 & 102.46 & 1077 & 75.08 & 782.75 & 77.7 & 633.62 & 86.18 \\
\hline
\end{tabular}

Table 2B. Descriptive results of distance traveled in retention and transfer tests

\begin{tabular}{lllll}
\hline \multirow{2}{*}{ Distance } & \multicolumn{2}{c}{ Retention } & \multicolumn{2}{c}{ Contextual transfer } \\
\cline { 2 - 5 } & Mean & SE & Mean & SE \\
Control & 942.52 & 125.28 & 903.50 & 139.23 \\
LI & 336.56 & 43.56 & 447.76 & 40.01 \\
MI & 235.81 & 22.54 & 243.06 & 24.20 \\
HI & 942.52 & 125.28 & 903.5 & 139.23 \\
\hline
\end{tabular}

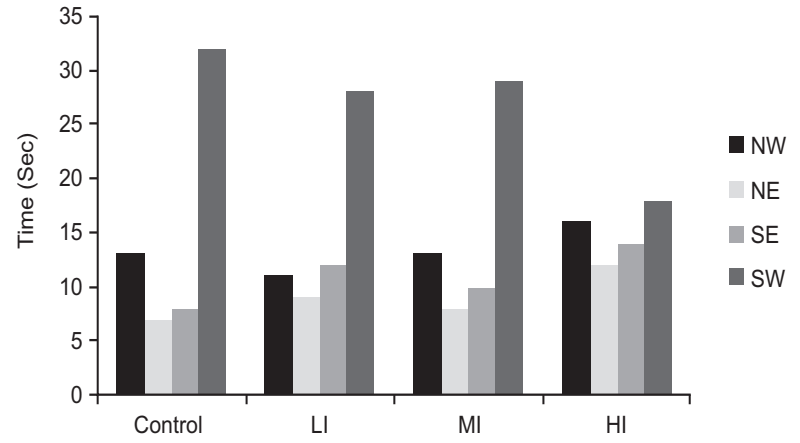

Figure 3. Time spent in the four virtual quadrants during the probe test. The white column represents the escape quadrant.

Acquisition in the training period and retention tests with high-intensity noise

The mean values of performance at the high-intensity noise level in the retention test are shown in Figures 1B and $2 \mathrm{~B}$. Significant differences were observed between the control and HI groups in both escape latency and distance traveled $\left(F_{3,28}=10.65, p<.05\right.$, and $F_{3,28}=11.01$, $p<.05$, respectively). The Tukey Honestly Significant Difference test revealed significant differences between the control, MI, and LI groups and HI group $(p<.05)$ in both latency and distance traveled, with no significant differences between the control and LI groups and MI group in either latency or distance traveled.

Acquisition in the training period and contextual transfer test with high-intensity noise immediately after exposure to 30 min continuous high-intensity noise

Significant differences were found in escape latency (Figure 1B) and distance traveled (Figure 2B) in the contextual transfer test (i.e., exposure to $30 \mathrm{~min}$ of continuous high-intensity noise; $F_{3,28}=7.62, p<.05$, and $F_{3,28}=7.55, p<.05$, respectively). This difference between the control group and LI and HI groups ( $p<$ .05 ) and between the LI and MI groups and HI group $(p<.05)$ in escape latency was significant, but no significant difference was found between the control and MI groups. Moreover, significant differences were found between the control, LI, and MI groups and HI group $(p<.05)$ in distance traveled, but the differences between the control group and LI and MI groups and between the LI group and MI group were not significant. 


\section{Discussion}

The present study tested the notion that continuous high-intensity noise may influence performance and learning in spatial memory tasks and that these effects are variable based on the contextual conditions (e.g., similar or dissimilar contexts between the training and tests). The idea that various intensities of noise during acquisition may cause different performance levels has been debated. The noise intensities we used were representative of the intensities emitted from sporting matches that produce a wide range of noise (52-105 dbA) (Gheraat et al., 2009).

An increase in noise intensity is hypothesized to impair performance in spatial tasks. Escape latency and distance traveled in the HI group were at the highest levels in the training period. Learning was expected to progress during the training days at all noise intensities, but this process was disrupted in the HI group, which may have been attributable to nonadaptation to the highintensity noise (Waye, 2003) or overarousal (YerkesDodson Law; Yerkes \& Dodson, 1908). These findings are consistent with the results of several previous studies (Glass \& Singer; Gheraat et al., 2009; Zhang et al., 2008; Stansfeld et al., 2005) that found a continuous improvement in performance during the training period. However, the HI group did not achieve comparable improvement in time and distance. The present results suggest that exposure to high-intensity noise causes learning impairments revealed by memory and visual tasks in infants (Wachs, Uzgiris, \& Hunt, 1971), resulting in learned helplessness, cognitive fatigue, and a consequent loss of information processing capacity (Hambrick-Dixon, 1986). These findings did not confirm the results of some previous investigations in which subjects adapted to the influence of noise during task activities by filtering out unwanted noisy stimuli (Haller et al., 2009) or the noise increased the dynamic range and ability of cochlear-implant subjects to encode temporal information (Zeng et al., 2000). In the visible test, no significant difference was found between the control, LI, and MI groups. But the most significant impairment was shown in the HI group. This test is intended to assess visuomotor coordination (Gheraat et al., 2009; Waye, 2003). High-intensity noise impairs visuomotor coordination in rats. In contrast, previous investigations did not find a significant decrease in performance on visible tests (Gheraat et al., 2009), tests of visual and intellectual function (Broadbent, 1979), or the separation of visual cues (Hambrick-Dixon, 1986) as a result of high-intensity noise. To learn the task adequately, the subject should be directed to rely on relevant visual cues rather than other sources of information. However, because of a disrupted learning process or imperfect transfer of learning (Maes \& de Groot, 2002) under high-intensity noise, rats were presumed to not be able to separate the appropriate and inappropriate information.

Results of the retention test with high-intensity noise were interesting. Although further impairment was expected to result from increasing the noise intensity, performance in the MI group was better than in the LI group. This finding is consistent with Gheraat et al. (2009) who found better results in the MI group than in the LI group in a Morris water maze task with intermittent noise. Another study found that children did not adapt to noise during a play period (i.e., retrieval; Hambrick-Dixon, 1986). In contrast, Glass \& Singer (1972) found that an equal context during training and testing (i.e., familiar noise) reflecting psychological and behavioral adaptation leads to better results. Additionally, the greatest latency and distance were recorded in the HI group, which may be attributable to a disruption of the learning process (Gheraat et al., 2009) or overarousal (Yerkes \& Dodson, 1908).

The main discussion of the present study is about the very interesting findings from the contextual transfer test after exposure to $30 \mathrm{~min}$ of continuous highintensity noise. Contrary to expectations, no significant difference was found between the control and MI groups during this test. However, significant differences were found between the LI and HI groups and control group. As shown in Figures 2A and 2B, significant differences were found between the retention and transfer tests only with regard to latency and distance traveled in the LI group, with no significant increases in the other groups. The highest escape latency and distance traveled were observed in the HI group, similar to the other tests. These findings support previous results indicating that listeners experience a perceptual advantage (i.e., a "release from masking") with interrupted noise than with continuous noise at equivalent signal-to-noise ratios (Stuart, 2005). Furthermore, higher-level working memory and the motor feedback response in a visual memory task significantly increased with continuous noise compared with conventional noise (Haller et al., 2009). These variations may be a function of the nature of the task (Hambrick-Dixon, 1986). These findings are inconsistent with Hambrick-Dixon's assertion that no significant difference exists between perceptual learning tasks (i.e., visual cues) in long-term compared with short-term high-intensity noise exposure (HambrickDixon, 1986). Moreover, another contrary theory states that noise increases the amount of energy applied to the primary task cues (Broadbent, 1979).

Finally, with regard to exposure to moderateintensity noise and its effects on performance in contextual transfer tests, no significant differences were found between performance on day 3 of the training period and retention in the contextual transfer test in the MI group. Exposure to moderate-intensity noise during the training period may present more meaningful 
learning compared with low-intensity noise. Therefore, exposure to $30 \mathrm{~min}$ of continuous high-intensity noise did not impair test results in the MI group. Indeed, this result may help instructors appropriately improve the learners' advantages from the training context. Further studies are needed to explore the role of various intensities and frequencies of noise during training, retention, and different transfer tests in human performance and learning.

\section{Acknowledgements}

We would like to acknowledge the Islamic Azad University, Ali Abad Katool Branch for financial support of this study. Also, all experiments conducted in this study were held at the Pasteur Institute of Iran, Department of Physiology \& Pharmacology under the supervision of Prof. Nasser Naghdi. Our appreciation is extended to Mr. R. Maes, Mr. S.A. Stansfeld, and Mr. J. Zhang for sending their articles and Mrs. E. Soleimannejad for her comments on the manuscript.

\section{References}

Akdogan, O., Selcuk, A., Take, G., Erdoğan, D., \& Dere, H., (2009). Continuous or intermittent noise exposure, does it cause vestibular damage? An experimental study. Auris Nasus Larynx, 36(1), 2-6.

Balmer, N. J., Nevill, A. M., Lane, A. M., Ward, P., Williams, A. M., \& Fairclaugh, S. H. (2007). Influence of crowd noise on soccer refereeing consistency in soccer. Journal of Sports Behavior, 30(2), $130-145$.

Bell, P. A., Hess, S., Hill, E., Kukas, S. L., Richards, R. W., \& Sargent, D. (1984). Noise and context-dependent memory. Bulletin of the Psychonomic Society, 22, 99-100.

Broadbent, D. E. (1979). Human performance and noise. In C. M. Harris (Ed.), Handbook of noise control (pp. 2066-2085). New York: McGraw-Hill.

Dimitrijevic, A., Michalewski, H. J., Zeng, F. G., Pratt, H., \& Starr, A. (2008). Frequency changes in a continuous tone: auditory cortical potentials. Clinical Neurophysiology, 119(9), 2111-2124.

Downey, P., \& Harrison, J. M. (1992). Effects of tone frequency and intensity on the auditory brainstem response (ABR) from albino and pigmented rats. Journal of the Experimental Analysis of Behavior, 59(2), 129-137.

Fiedler, F. E., \& Fiedler, J. (1975). Port noise complaints: Verbal and behavioral reactions to airport-related noise. Journal of Applied Psychology, 60(4), 498-506.

Gheraat, M. A., Naghdi, N., Jalaei, B., Mokhtari, P., Rezaei Moghadam, F., Soleimannejad, E., \& Eidi Abarghani, L. (2009). Impairment of spatial performance by environmental noise. Scholarly Research
Exchange, 2009, 840620

Glass, D. C., \& Singer, J. E. (1972). Urban stress: Experiments on noise and social stressors. New York: Academic Press.

Haller, S., Homola, G. A., Scheffler, K., Beckmann, C. F., \& Bartsch, A. J. (2009). Background MR gradient noise and non-auditory BOLD activations: A data-driven perspective. Brain Research, 1282, 74-83.

Hambrick-Dixon, P. J. (1986). Effects of experimentally imposed noise on task performance of black children attending day care center near elevated subway trains. Developmental Psychology, 22(2), 259-264.

Jepson, P. D., Arbelo, M., Deaville, R., Patterson, I. A., Castro, P. (2003). Gas-bubble lesions in stranded cetaceans. Nature, 425, 575576.

Maes, J.H.R., \& de Groot, G. (2002). Effects of noise on the performance of rats in an operant discrimination task. Behavioural Processes, 61(1), 57-58.

Malakoff, D. (2001). A roaring debate over ocean noise. Science, 291(5504), 576-578.

Naghdi, N., Rezaei, M., \& Fathollahi, Y. (2006). Microinjection of ritanserin into the CA1 region of hippocampus improves scopolamine-induced amnesia in adult male rats. Behavioral Brain Research, 168, 215-220.

Nevill, A. M., Balmer, N. J., \& Williams, A. M. (2002). The influence of crowd noise and experience upon refereeing decisions in football. Psychology of Sport and Exercise, 3(4), 261-272.

Schmidt, R. A. (1999). Motor control and learning: A behavioral emphasis, $3^{\text {rd }}$ edition (pp. 111-127). Champaign, IL: Human Kinetics.

Stansfeld, S. A., Berglund, B., Clark, C., Lopez-Burrio, I., Fischer, P., Ohrstrom, E., Haines, M. M., Head, J., Hygge, S., van Kamp, I., \& Berry, B. F. (2005). Aircraft and road traffic noise and children's cognition and health: A cross-national study. Lancet, 365(91475), 1942-1999.

Stuart, A. (2005). Development of auditory temporal resolution in school-age children revealed by word recognition in continuous and interrupted noise. Ear and Hearing, 26(1), 78-88.

Syka, J., \& Popelar, J. (1982). Noise impairment in the guinea pig: I. Changes in electrical evoked activity along the auditory pathway. Hearing Research, 8(3), 263-272.

Talpalery, A. E., \& Grossman, Y. (2005). Sonar versus whales: Noise may disrupt neural activity in deep-diving cetaceans. Undersea and Hyperbaric Medical Society, 32(2), 135-139.

Wachs, T. D., Uzgiris, I. C., \& Hunt, J. M. (1971). Cognitive development in infants of different age levels and from different environmental backgrounds: An explanatory investigation. Merrill-Palmer Quarterly: Journal of Developmental Psychology, 17(4), 283-317.

Waye, G., Clow, A., Edwards, S., Hucklebridge, F., \& Rylander, R. (2003). Effects of nighttime low frequency noise on the cortisol response to awakening and subjective sleep quality. Life Sciences, 72(8), 863-875.

Yerkes, R. M., \& Dodson, J. D. (1908). The relation of strength of stimulus to rapidity of habit-formation. Journal of Comparative Neurology and Psychology, 18, 459-482.

Zeng, F. G., Fu, Q. J., \& Morse, R. (2000). Human hearing enhanced by noise. Brain Research, 869, 251-255.

Zhang, J., Chen, L., Gao, F., Pu, Q., \& Sun, X. (2008). Noise exposure at young age impairs the auditory object exploration behavior of rats in adulthood. Physiology and Behavior, 95(1-2), 229-234. 